\title{
Endoscopic Endonasal Cerebrospinal Fluid Leak Repair on the Ventral Midline Skull Base: A Single Neurosurgical Center Experience
}

\author{
Cihan ISLER, Merdin Lyutviev AHMEDOV, Mehmet Yigit AKGUN, Baris KUCUKYURUK, Nurperi GAZIOGLU, \\ Galip Zihni SANUS, Necmettin TANRIOVER \\ Istanbul University, Cerrahpasa Medical Faculty, Department of Neurosurgery, Istanbul, Turkey
}

\section{ABSTRACT}

AIM: To present the results of endoscopic endonasal repair of ventral midline skull base cerebrospinal fluid (CSF) leak (VMSBL) at our institution and to discuss the technique and results from a neurosurgical perspective.

MATERIAL and METHODS: A retrospective analysis of all VMSBL cases that underwent endoscopic endonasal skull base approach (EESBA) for CSF leak repair at a single tertiary neurosurgical center was performed. Twenty six patients with an average age of 44.4 (range: 17-63) years were included in the study.

RESULTS: The etiology of VMSBL was spontaneous in 16 patients, traumatic in 7, and iatrogenic in 3 . The leakage site was the cribriform plate in 13 patients, ethmoidal cells in 7 , and sphenoid sinus in 3 . There were multiple leaks in 3 patients. This approach for VMSBL repair was performed 28 times on 26 patients. The success rate was $88.5 \%$ (23/26 patients) after primary endoscopic repair and $96 \%$ after the second attempt. The location of the leakage site relative to the upper attachment of the middle turbinate played a crucial role in the anteriorly located VMSBL, which made an impact on the surgical repair plan. All the 16 cases with accompanying meningoencephaloceles were treated successfully by EESBA. Use of vascularized pedicled flaps to support the repair site resulted in $100 \%$ success after primary repair.

CONCLUSION: EESBA is safe and highly effective and can be a first-line surgical treatment option for VMSBL. In addition, it enables adequate reconstruction of ventral midline skull base meningoencephaloceles regardless of size and location.

KEYWORDS: Cerebrospinal fluid leak, Endoscope, skull base, Ventral midline, Endonasal approach

\section{INTRODUCTION}

$\mathrm{T}$ The last two decades have witnessed a smooth transition from open to endoscopic approaches in the management of skull base cerebrospinal fluid (CSF) leaks $(7,10,18,19,22,29)$. A connection to the subarachnoid space, most likely associated with a disruption of the arachnoid and dura mater with accompanying bone defect, is a prerequisite for CSF leak and inevitably carries a risk for ascending infection to the intracranial space $(15,25,26,31)$.
CSF leaks have been grouped into three distinct etiologic categories, namely, traumatic, spontaneous, and iatrogenic (due to previous operation). Furthermore, each group has been subdivided into acute and delayed types (6). The first line of treatment is surgery in all cases of spontaneous etiology and in most cases of iatrogenic cause of skull base CSF leak $(36,38)$. On the contrary, initial conservative management has been advocated for traumatic CSF leaks $(8,12)$. When conservative management fails, usually at the end of two weeks, surgical management of rhinorrhea is mandatory in traumatic cases $(16,28)$. 
The CSF may leak through any part of the ventral midline skull base, including the cribriform plate, as well as the frontal, ethmoid, and sphenoid sinuses $(3,23)$. Spontaneous CSF leak and meningoencephaloceles within the lateral recess of the sphenoid sinus is a different entity that requires a distinct surgical approach (21). Due to the overall low success rates of craniotomy for CSF leaks located in the ventral midline and laterally and the possibility of associated intracranial complications, endoscopic endonasal reconstruction has gradually succeeded in replacing the traditional transcranial approaches $(16,33)$. Although replacing the transcranial approaches by the extracranial approach has a long history $(13,24,30,34,37)$, general consensus on the most appropriate endoscopic endonasal surgical technique with regard to the type of graft and use of vascularized flaps has not been reached.

In this study, our aim was to present the results of endoscopic endonasal repair of ventral midline skull base CSF leaks (VMSBL) at our institution since 2008 and to discuss the technique and results from a neurosurgical point of view in light of the current literature.

\section{- MATERIAL and METHODS}

There were 39 patients who underwent 43 endoscopic endonasal skull base approach (EESBA) for repair of CSF fistula between September 2008 and December 2016 at the Cerrahpasa Medical Faculty, Department of Neurosurgery. Patients with CSF leaks located at the lateral recess of the sphenoid sinus $(n=13)$ were excluded from this study because our aim was to present the surgical technique and results for VMSBL. Furthermore, the surgical technique for lateral recess leaks is different and many cases need a lateral extension of an endoscopic endonasal skull base route, including a minimal transpterygoid approach to reach the leak site. The results for these cases will be presented in a separate study.

Biochemical analyses of the CSF were made by $\beta 2$ transferrin assay. Fine-cut (1-mm thickness) high resolution computed tomography (HRCT) scan through the skull base was performed on all 26 patients prior to surgery. Magnetic resonance cisternography (MRc) was performed on all patients with active CSF leak $(n=25)$ in order to confirm the diagnosis and location of the leak and to design the surgical repair plan. Informed consent was obtained from each patient.

\section{- RESULTS}

This study included 26 patients with VMSBL; the average age was 44.4 years (range, 17-63 years), 18 were women and 8 were men. The mean follow-up period of patients was 48.15 months (range, 3-98 months). The etiologies of CSF leak were spontaneous in 16 patients, traumatic in 7 patients, and iatrogenic in 3 patients who had undergone previous functional endoscopic sinus surgery elsewhere.

The demographic data of the study population are listed in Table I. The leakage site was the cribriform plate around the crista galli in 13 patients, anterior ethmoidal cells in 3, posterior ethmoidal cells in 4 , and sphenoid sinus in 3 patients. There were multiple leakage sites in 3 additional patients and all had sphenoid sinus involvement, in addition to the posterior ethmoidal cells $(n=2)$, and crista galli $(n=1)$. The duration of the CSF leak varied from 2 weeks to 10 years. In all cases that underwent MRc, the leakage sites were identified successfully.

In the 26 patients, 28 EESBAs were performed for the repair of VMSBL. Of the 23 patients who underwent a single successful endoscopic operation at our institution, 2 patients had history of failed transcranial repairs elsewhere. One patient in this series required a second endoscopic surgery in order to resolve a persistent leak. Another patient operated upon during the learning curve of the skull base endoscopy needed a transcranial approach following the initial endoscopic endonasal attempt. The last patient required a transcranial approach after two failed endoscopic surgeries. Overall, the success rate of primary endoscopic repair for this series was $88.5 \%(23 / 26)$ and the rate increased to $96 \%$ on the second attempt (Table II).

A mononostril endoscopic route was used in 16 of 26 patients, whereas a binostril approach was the choice in 10 of 26 patients. The mononostril access was preferred when the site of CSF leak was located anteriorly (i.e., crista galli, fovea ethmoidalis, and anterior ethmoidal cells) and rhinorrhea was through only one nostril. Of the 16 patients with CSF leakage through the crista galli and/or anterior ethmoidal cells, 14 patients were operated through the mononostril endoscopic route. On the other hand, the binostril approach was the choice for the majority of cases with CSF leak in the sphenoid sinus and/or posterior ethmoidal cells.

\section{Surgical Considerations}

Based on the intraoperative direct endoscopic visualization, the 13 patients who had leakage at the cribriform plate around the crista galli were divided into medial (through the cribriform plate) and lateral (through the fovea ethmoidalis) sites according to the location of the leakage site in relation to the upper attachment of the middle turbinate. In all cases, the borders of all anterior bony defects were identified prior to graft placement in order to stimulate osteogenesis. The anteriorly located small defects were closed with onlay (overlay) free muscle/fascial grafts, which were usually harvested from the abdomen. The middle turbinate was used as a vascularized flap for lateral leaks (through fovea ethmoidalis), whereas medialization of the middle turbinate alone was adequate for medial leaks through the cribriform plate. We used fibrin glue in order to increase the biomechanical strength of CSF leak repair and augment graft adherence (9).

Overall, relatively small defects $(n=12)$ were closed with a combination of onlay free fat, fascia, and muscle grafts. Larger defects $(n=4)$ were closed with an inlay abdominal fascia, with an overlying bone graft in some cases. A 4.0 vicryl suture was secured on the center of the inlay fascia graft to allow the necessary withdrawal following intracranial introduction; this technique needs care to avoid inadvertent compression of neurovascular structures. In three patients, pedicled middle turbinate flaps were used to cover the bony defect. We began to use vascularized pedicled nasoseptal flaps to support the 
Table I: Demographic Data of Patients with Ventral Midline Skull Base Cerebrospinal Fluid Leak

\begin{tabular}{|c|c|c|c|c|c|}
\hline $\begin{array}{l}\text { Patient } \\
\text { number }\end{array}$ & Age (years)/ Sex & Etiology & Leakage site & $\begin{array}{l}\text { Encephalocele } \\
\text { formation }\end{array}$ & $\begin{array}{c}\text { Number of operations } \\
\text { E/TC (Total) }\end{array}$ \\
\hline 1 & $39 / M$ & spontaneous & CG & Yes & $E(1)$ \\
\hline 3 & $52 / F$ & spontaneous & EP & Yes & $E(1)$ \\
\hline 6 & $45 / M$ & traumatic & EA & No & $E(1)$ \\
\hline 7 & $60 / M$ & iatrogenic & EP & Yes & $E(1)$ \\
\hline 8 & $17 / F$ & iatrogenic & CG & No & E/E/TC (3) \\
\hline 12 & $45 / F$ & traumatic & EP & Yes & $E(1)$ \\
\hline 13 & $52 / F$ & spontaneous & EP & No & $E(1)$ \\
\hline 14 & $46 / M$ & traumatic & $S S+E P$ & Yes & TC / E (2) \\
\hline 15 & $63 / F$ & spontaneous & CG & Yes & $E(1)$ \\
\hline 16 & $61 / M$ & traumatic & SS & No & $E(1)$ \\
\hline 17 & $55 / \mathrm{F}$ & spontaneous & CG & No & $E(1)$ \\
\hline 18 & $39 / F$ & traumatic & $\mathrm{SS}+\mathrm{CG}$ & Yes & $E(1)$ \\
\hline 24 & $63 / F$ & spontaneous & SS & Yes & $E(1)$ \\
\hline 25 & $40 / F$ & spontaneous & CG & Yes & $E(1)$ \\
\hline 26 & $23 / F$ & spontaneous & CG & Yes & $E(1)$ \\
\hline
\end{tabular}

CG: Crista galli, SS: Sphenoid sinus, EP: Ethmoid posterior, EA: Ethmoid anterior, E: Endoscopic endonasal, TC: Transcranial, F: Female, M: Male.

repair site within the last four years, and seven patients, in this series, required vascularized nasoseptal flap for successful primary repair of the leakage.

Sixteen patients in this series had intraoperatively confirmed meningoencephaloceles and all were identified through preoperative magnetic resonance imaging (MRI) and MRc. In these cases, the etiologies of CSF leak were spontaneous in 12, traumatic in 3 , and iatrogenic in 1 . Only half of these patients $(n=8)$ with meningoencephaloceles required a pedicled middle turbinate or nasoseptal flap to prevent herniation of cerebral tissue. When a small meningoencephalocele was present and an elevated intracranial pressure was suspected, the lesion was reduced with bipolar coagulation (35). Larger meningoencephaloceles were excised from the pedicle prior to the repair of the dural defect; this was followed by circumferential removal of the mucosa. Some of these cases with large meningoencephaloceles needed an additional vascularized flap, since the free fascia graft was expected to decrease in size by about $20 \%$ postoperatively $(14,35)$. All patients with existing meningoencephalocele were treated successfully by the endoscopic endonasal approach. 
Importantly, a forced inspiration maneuver under direct endoscopic view was performed by the anesthesiologist to ensure that the seal was intact at the end of the operation.

No major peri- and postoperative, intra- and extracranial complications occurred during the follow-up period. Although two of our patients had meningitis prior to surgery, none developed any form of infection, such as meningitis or brain abscess during the postoperative period. Mean hospital stay was 8.65 days (range, 3-25 days). In all patients, lumbar catheter for postoperative CSF drainage was placed in the operating room under general anesthesia; however, CSF pressure was not monitored. We have routinely used lumbar CSF drainage for an average of three days in the postoperative period to facilitate the wound healing at the leakage site.

\section{Representative Cases}

Case 1: A 61-year-old man sustained head trauma due to a traffic accident and presented to our clinic with late-

Table II: Surgical Data and Outcomes of Patients with Ventral Midline Skull Base Cerebrospinal Fluid Leak

\begin{tabular}{|c|c|c|c|c|}
\hline Patient & Endonasal route & Surgical graft & $\begin{array}{c}\text { OUTCOME } \\
\text { endoscopic surgery }\end{array}$ & $\begin{array}{c}\text { OUTCOME } \\
2^{\text {nd }} \text { endoscopic surgery } \\
\end{array}$ \\
\hline 1 & M & $\mathrm{FL}$ & Successful & \\
\hline 2 & B & $\mathrm{FL}+\mathrm{F}$ & Successful & \\
\hline 3 & B & $\mathrm{FL}+\mathrm{MT}$ & Successful & \\
\hline 4 & B & $\mathrm{F}$ & Unsuccessful & Successful \\
\hline 5 & M & $\mathrm{FL}+\mathrm{M}$ & Unsuccessful & \\
\hline 6 & B & $\mathrm{FL}+\mathrm{F}+\mathrm{M}$ & Successful & \\
\hline 7 & B & $A F+F+M$ & Successful & \\
\hline 8 & M & $A F+F+M+B o$ & Unsuccessful & Unsuccessful \\
\hline 9 & M & $A F+F+M+B o$ & Successful & \\
\hline 10 & M & $A F+F+M$ & Successful & \\
\hline 11 & M & $A F+F+M+B o$ & Successful & \\
\hline 12 & M & MT & Successful & \\
\hline 13 & M & $\mathrm{AF}+\mathrm{M}$ & Successful & \\
\hline 14 & B & $A F+F+N S F$ & Successful & \\
\hline 15 & B & $A F+M+N S F$ & Successful & \\
\hline 16 & B & $A F+F+M+M T+N S F$ & Successful & \\
\hline 17 & M & $A F(I N L A Y)+F+M$ & Successful & \\
\hline 18 & B & $A F+F+M+M T+N S F$ & Successful & \\
\hline 19 & M & $\begin{array}{c}A F+F+M+M T+N S F \\
+B_{0}\end{array}$ & Successful & \\
\hline 20 & M & $A F+M+M T+N S F$ & Successful & \\
\hline 21 & M & $A F+F+M+M T$ & Successful & \\
\hline 22 & M & $A F+F+M$ & Successful & \\
\hline 23 & M & $A F+F+M$ & Successful & \\
\hline 24 & B & $A F+F+M+N S F$ & Successful & \\
\hline 25 & M & $A F+F+M$ & Successful & \\
\hline 26 & M & $A F+F+M$ & Successful & \\
\hline TOTAL & & & $88.5 \%$ SUCCESS & 96\% SUCCESS \\
\hline
\end{tabular}

B: Binostril, M: Mononostril, FL: Fascia lata, F: Abdominal fat, M: Abdominal muscle, MT: Middle turbinate, AF: Abdominal fascia, NSF: Nasoseptal flap, Bo: Bone. 
onset rhinorrhea five days after. HRCT scan through the skull base revealed a clival defect with soft tissue density protruding into the sphenoid sinus. MRc confirmed a CSF leak through the clival defect into the sphenoid sinus with an accompanying meningocele (Figure 1A-E). He underwent binostril endoscopic endonasal transsphenoidal resection of the meningocele and repair of the defect on the upper one third of the clivus just inferior to the posterior clinoid processes with inlay muscle and fascia grafts. The free grafts were supported with vascularized nasoseptal flap, abdominal fat in the sphenoid sinus, and fibrin glue (Figure 2A-H). Finally, a $16 \mathrm{~F}$ Foley catheter was placed within the nasal cavity in order to facilitate reconstruction. CSF was drained by an indwelling spinal catheter and antibiotics were continued for five days postoperatively. The CSF leak resolved instantly following the endoscopic endonasal surgery.

Case 2: A 52-year-old man presented with spontaneous nasal discharge of clear fluid for four months. Diagnostic work-up included CSF analysis, which was positive for $\beta 2$ transferrin. HRCT scan demonstrated a bony defect on the cribriform plate around the crista galli on the left, lateral to the upper attachment of the middle turbinate and coinciding with the location of the fovea ethmoidalis. MRc confirmed a CSF leak through the fovea ethmoidalis and sufficiently indicated an accompanying encephalocele (Figure $3 \mathrm{~A}-\mathrm{H}$ ).

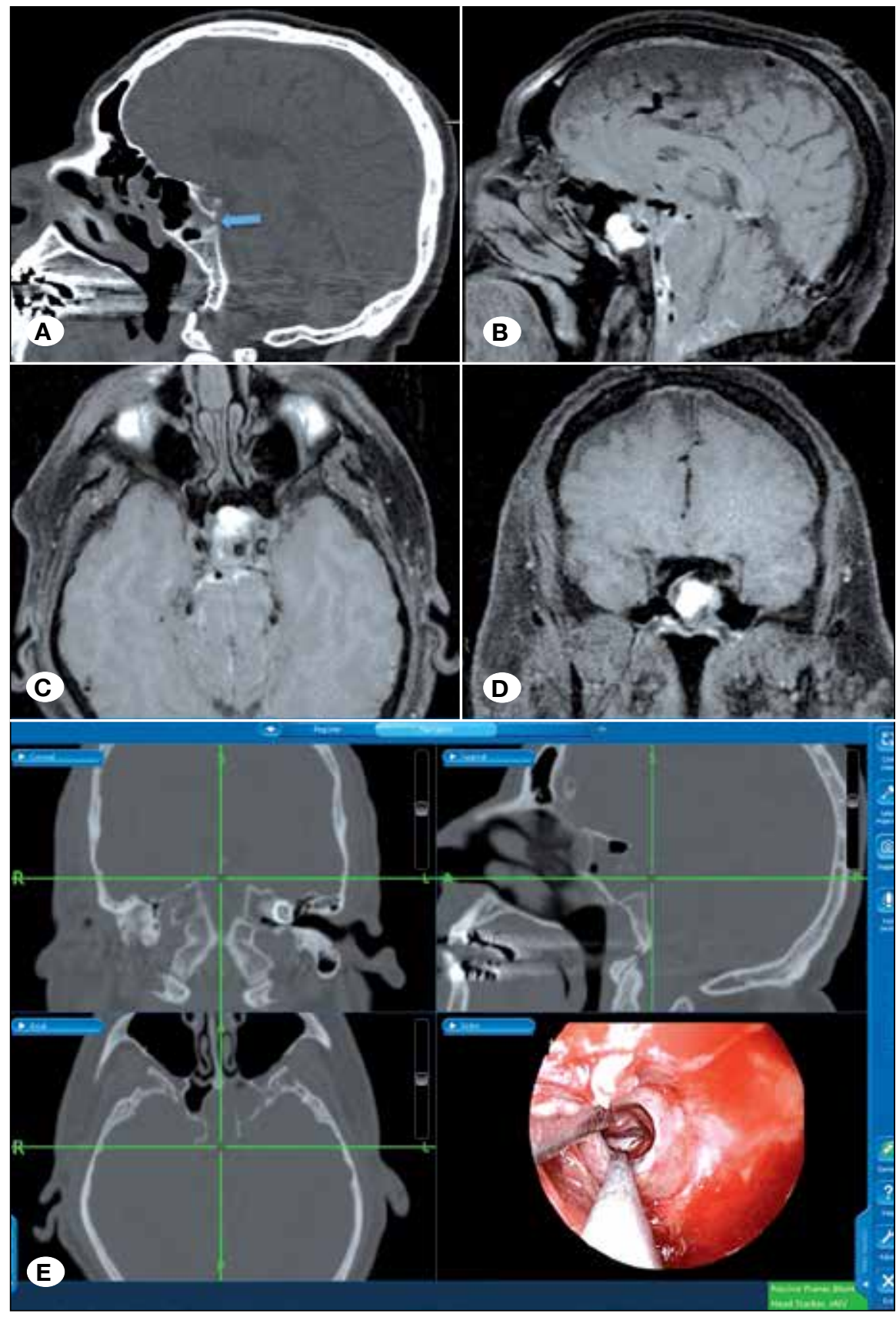

Figure 1: Radiologic examinations in representative case 1.

A) Preoperative sagittal computed tomography image shows a soft tissue density protruding through the clival defect (blue arrow) on the posterior part of the sphenoid sinus. B-D) Preoperative sagittal-, axial-, and coronal-plane magnetic resonance cisternography shows cerebrospinal fluid leakage through the clival defect. E) Intraoperative neuronavigation indicates the clival defect through which the pons and transverse pontine veins are seen. 
Through a mononostril approach, the meningoencephalocele was reduced with bipolar coagulation and the defect was repaired using abdominal muscle, fat, and fascia free grafts along with fibrin glue. Finally, the middle turbinate was used as a vascularized flap and was rotated laterally towards the supporting tissue, followed by placement of a $16 \mathrm{~F}$ Foley catheter. Although the CSF leak stopped immediately after the operation, the CSF drainage through an indwelling spinal catheter and antibiotic prophylaxis were continued for a couple of days. The postoperative course was uneventful and the patient was discharged on postoperative day 8 .

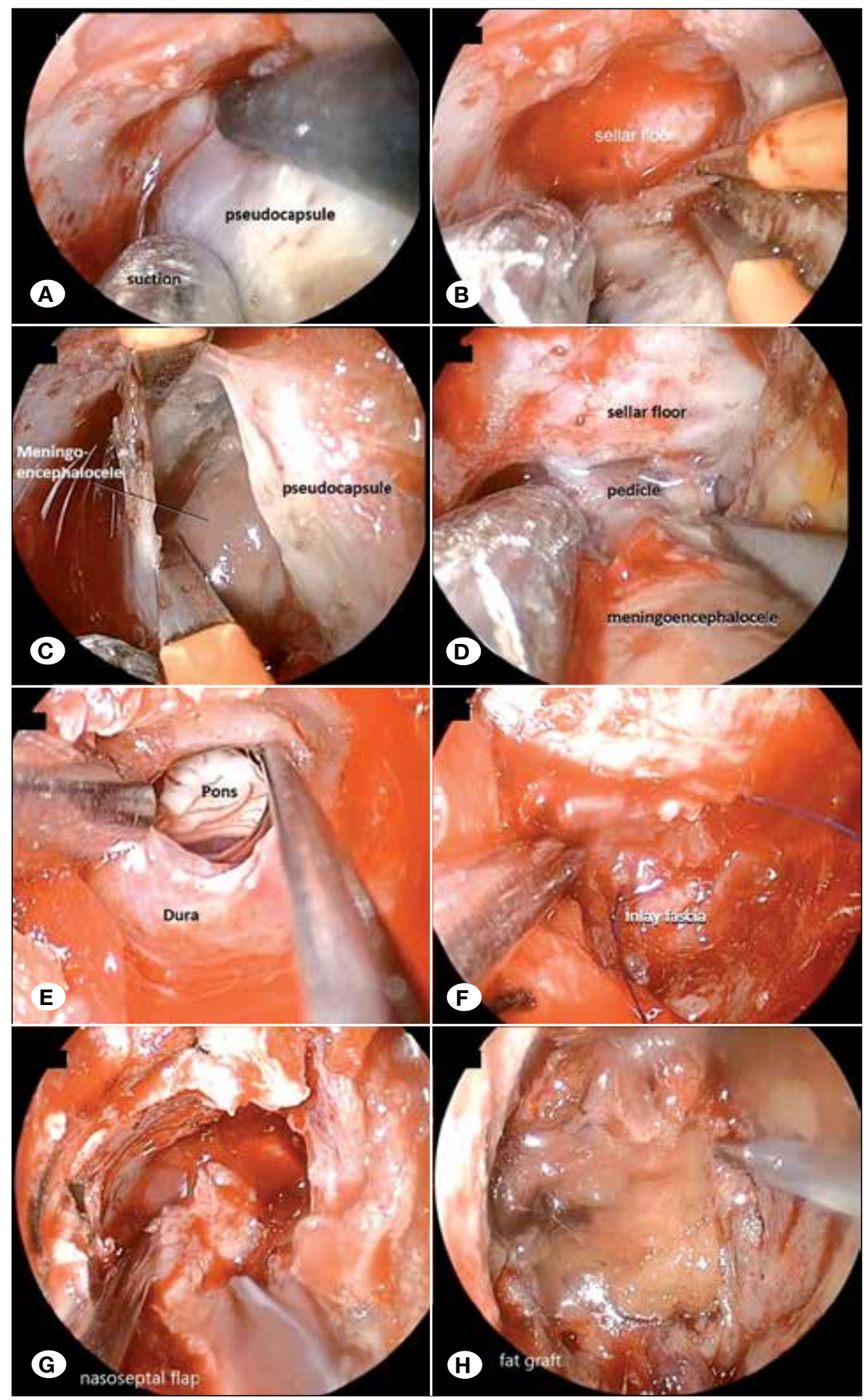

Figure 2: Intraoperative endoscopic images in representative case 1.

A) Cerebrospinal fluid is seen through the pseudocapsule of the meningocele in the sphenoid sinus. B) The sellar floor is exposed via bipolar coagulation and partial reduction through the superior edge of the pseudocapsule. C) Opening the pseudocapsule reveals the meningocele. D) The pedicle of the meningoencephalocele at the clival defect becomes prominent.

E) The meningocele is removed and the transverse pontine vein and the pons are exposed through the dural defect. F) A 4.0 vicryl-secured fascia is inserted into the defect. G) A vascularized pedicled nasoseptal flap is reflected on to the upper clival defect to support the closure with fascia. H) Free fat graft and fibrin glue are used at the end of the reconstruction. 
Case 3: A 63-year-old woman presented with intermittent spontaneous nasal discharge of clear fluid for one year. $\beta 2$ transferrin assay of the fluid was positive. MRc could not be performed in this patient due to claustrophobia. Instead, intrathecal contrast-enhanced computed tomography (CT) cisternography revealed the probable location of the CSF leak and encephalocele at the anterosuperior edge of the sphenoid sinus along the anterior portion of the planum sphenoidale.
Through a binostril endoscopic endonasal transsphenoidal approach, the sphenoid sinus was opened to expose the sellar floor, the bilateral cavernous carotid protuberances and optic canals, and the opticocarotid recesses. The leakage site and the meningoencephalocele were identified by defining the tuberculum sellae and following the planum anteriorly towards the anterosuperior edge of the sphenoid sinus. The meningoencephalocele was located on the right just lateral
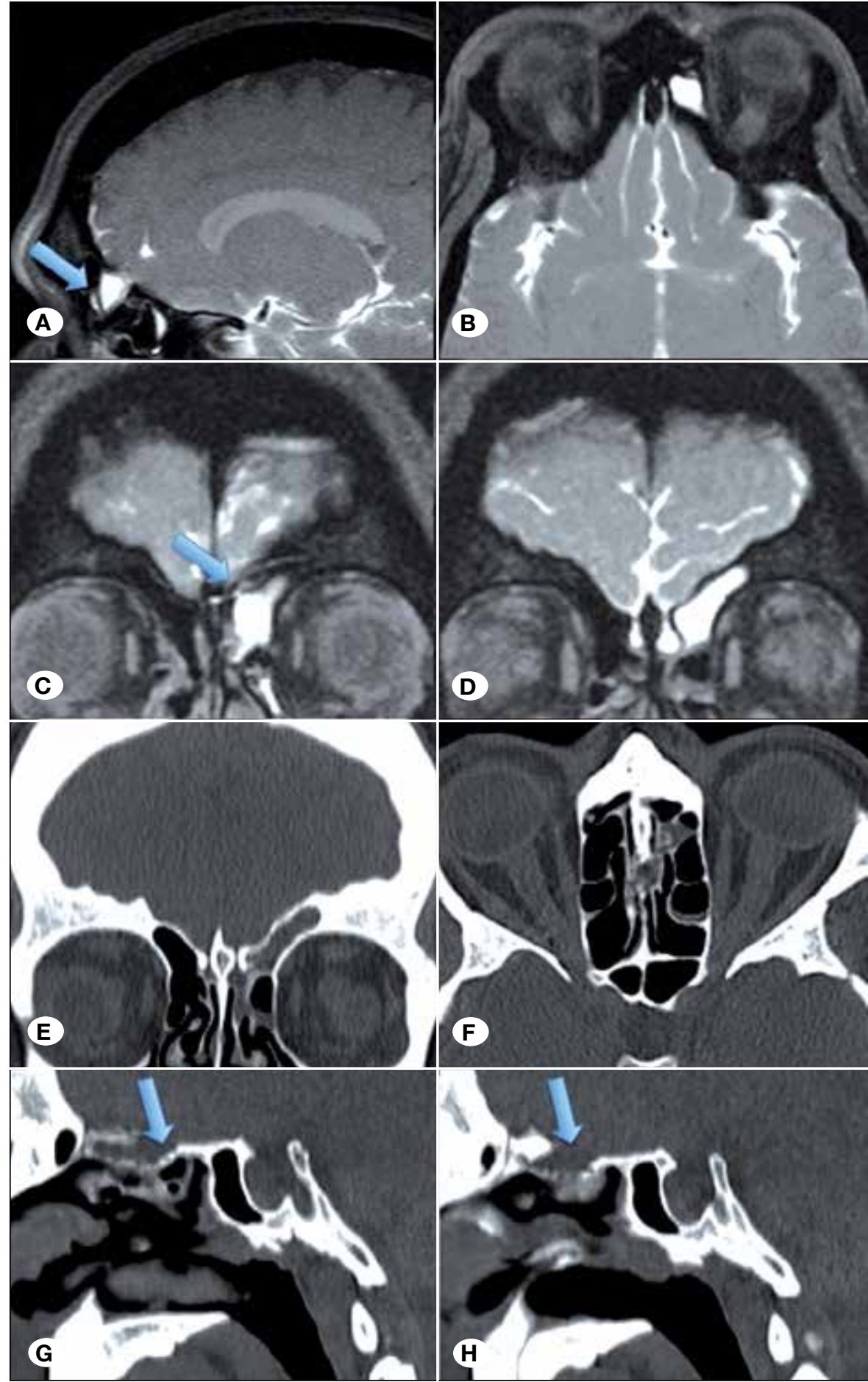

Figure 3: Preoperative radiologic examinations in representative case 2. A-D) Magnetic resonance cisternography in three planes shows meningoencephalocele formation and cerebrospinal fluid leakage (blue arrows in A and C). E-H) Computed tomography scan reveals thinning and a defect in the fovea ethmoidalis with encephalocele formation (blue arrows in $\mathrm{G}$ and $\mathrm{H}$ ). 
to an intrasphenoidal septum along the anterior part of the planum sphenoidale. Prior to the repair of the dural defect, the meningoencephalocele was reduced by bipolar coagulation and was excised from its pedicle, followed by circumferential removal of the mucosa. The defect was repaired with onlay muscle and abdominal fascia grafts, which were supported by vascularized nasoseptal flap, fat, and fibrin glue (Figure 4A-H). Then, a 16F Foley catheter with its inflated balloon was placed in the nasal cavity for two days. CSF drainage and antibiotic prophylaxis were continued for five days postoperatively. The rhinorrhea ceased in the immediate postoperative period and the patient had an uneventful recovery.

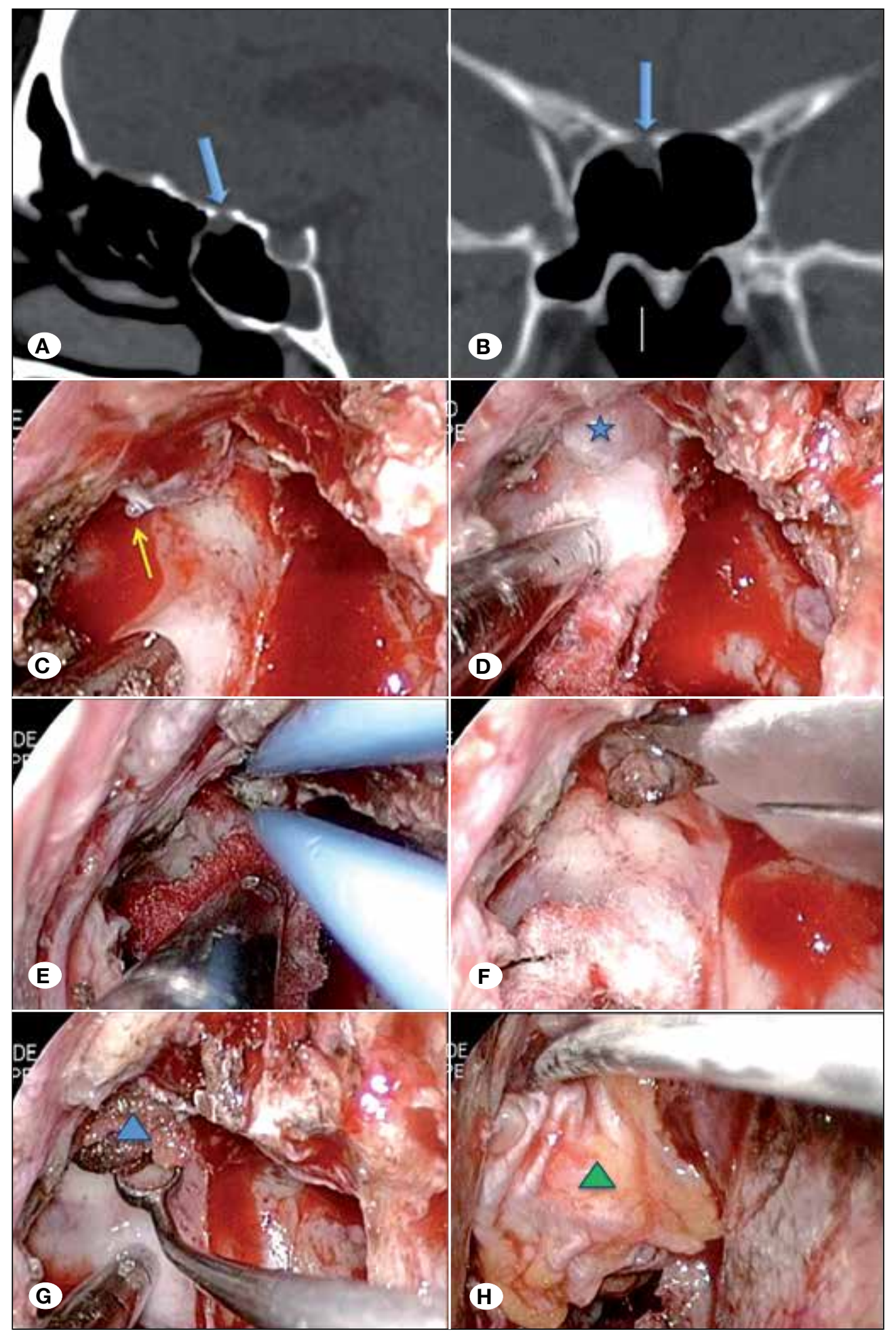

Figure 4: Representative case 3. A-B) Preoperative computed tomography scan shows a skull base defect with a meningoencephalocele that protrudes into the sphenoid sinus (blue arrows). Intraoperative findings show C-D) a drop of cerebrospinal fluid through the meningoencephalocele (yellow arrow), which protrudes into the sphenoid sinus (blue star). E) Bipolar coagulation and $\mathbf{F}$ ) excision of the meningoencephalocele using endoscopic scissors. G) A free onlay muscle flap (blue triangle) is used to cover the defect and H) free fascia graft (green triangle) is used to cover the nasoseptal flap along with fibrin glue. 


\section{DISCUSSION}

Har-el grouped CSF leaks as traumatic and non-traumatic (spontaneous) and further subdivided the traumatic group into surgical (iatrogenic) and non-surgical causes, based on a previous classification by Cairns $(6,11)$. In this study, however, we have grouped the etiology of VMSBLs in accordance with the three-tier system. The majority of our cases with CSF leak comprised spontaneous (62\%), followed by traumatic $(27 \%)$ and iatrogenic $(11 \%)$ causes. Some recent studies emphasized trauma as the most common cause of CSF leaks (32), whereas most of the available meta-analyses in literature cited spontaneous etiology as the most common (27).

Rhinorrhea may cause lethal infections and must be managed promptly and properly, beginning with the diagnosis. In some cases, the diagnosis of CSF leak through the ventral skull base may be challenging because of difficulties in analyzing the fluid and dilemmas in demonstrating the leak site by radiologic examinations. In all of our cases, HRCT was performed after confirmation of CSF leak by $\beta 2$ transferrin assay. HRCT was helpful not only for defining small-sized bony defects and fractures at the skull base, but also for intraoperative neuronavigation using serial images (2). MRc was performed in all VMSBL cases, except in one patient with severe claustrophobia, and guided us for the surgical planning. Intrathecal contrast-enhanced MRc identified the leakage site in all of our cases; this observation was consistent with the previously reported $100 \%$ sensitivity of this test in detecting CSF fistula (1). In addition, MRc allowed delineation of an accompanying encephalocele, including its size and the location of its pedicle relative to the skull base. We did not use intrathecal fluorescein in any of our cases since it is currently an off-label indication for intraoperative diagnosis of CSF leaks. Although it could be a valuable tool for endoscopic detection of intraoperative CSF leak, we believe that it was not a prerequisite for effective reconstruction of VMSBLs. Similar to previous reports, this study supported the high efficacy of endoscopic endonasal surgery for VMSBL without intrathecal fluorescein use (8).

Systematic reviews on endoscopic repair of CSF leaks have described the ethmoidal roof and the cribriform region as the most frequently involved sites $(27,32)$. Accordingly, crista galli was the most common site in our series (48.2\%), followed by the posterior ethmoidal cells $(20.7 \%)$, the sphenoid sinus (20.7\%), and the anterior ethmoidal cells (10.4\%). Correlation between the etiology and the leak site revealed a tendency for spontaneous VMSBLs to involve the crista galli (10/16). This finding was consistent with the results of the study of Lanza et al. on 36 patients (17). In another review, the cribriform plate, along with the lateral recess of the sphenoid sinus, was the most common site in patients with spontaneous idiopathic CSF leak (38).

The long history of extracranial approach to CSF leaks began in real terms with Oskar Hirsch's transseptal route to repair intrasphenoidal leaks. CSF fistulas on the dorsum sellae of two pituitary adenoma cases could not be reached by intracranial approach and Hirsch used mucosal flaps from the nasal septum to seal the leak sites through the sphenoid sinus. This was the first successful report on endonasal repair of CSF fistula on the skull base (13). Almost thirty years later, a group of otorhinolaryngology surgeons documented the first successful endoscopic endonasal CSF leak repair (37). Wigand, in fact, introduced the use of the endoscope through the nasal route in 1981. Since then, continuous improvements of the endoscopic system, along with gradual evolution of the technique, provoked a paradigm shift in the management of CSF fistulas from invasive open approaches to less invasive endoscopic procedures. Indeed, it has been proven that endoscopic endonasal repair of CSF leaks has a definite superiority over craniotomy; is less invasive than the transcranial approach; and is related with less morbidity and shorter length of hospital stay $(5,15,27)$. Furthermore, endoscopic repair is more advantageous in preserving olfaction and cosmetics.

A meta-analysis demonstrated that successful complete closure of endoscopic CSF leak was $90 \%$ on the average on the first attempt and increased to $96 \%$ after the second attempt. The reported success rates ranged from $60 \%$ to $100 \%(12,27,32)$. In our series of 26 patients with VMSBL, the primary success rate of endoscopic endonasal approach was $88.5 \%$ on the first attempt and increased to $96 \%$ after the second operation. Repair of CSF fistula failed on the first attempt in three patients; the respective sites of the leaks in these patients were the crista galli, anterior ethmoidal cells, and at multiple leakage sites on the sphenoid sinus and posterior ethmoidal cells. The resolution of the CSF leak in the patient with multiple leakage sites occurred following the second endoscopic attempt. The two remaining patients with persistent leaks underwent transcranial surgery, which were successful. These failed endoscopic operations occurred during the initial learning curve period of our endoscopic skull base surgery experience.

The relationship between the leakage site and the upper attachment of the middle turbinate may play a crucial role in anteriorly located VMSBLs. In this regard, anterior CSF leaks in our series had two distinct anatomic skull base targets. These comprised: 1) the crista galli region, specifically the horizontal cribriform plate (lamina cribrosa) of the ethmoid bone, which was laterally bound by the vertical lateral lamella and had a superior attachment to the middle turbinate; and 2) the fovea ethmoidalis of the frontal bone, which separates the ethmoidal cells from the anterior cranial fossa and is more lateral to the lateral lamella. The leakage sites were located medially in eight cases and through the fovea ethmoidalis in five cases. This compartmentalization of the leakage site based on the relationship with the upper attachment of the middle turbinate made an impact on the surgical repair plan. The middle turbinate was used as a vascularized flap and was rotated laterally in most lateral leaks through the fovea. In contrast, medial displacement of the middle turbinate was sufficient to support the onlay grafts for the medially located leaks through the horizontal cribriform plate (lamina cribrosa) of the ethmoid bone.

In this study, all 16 patients with accompanying meningoencephaloceles were successfully treated by EESBA. Only half of these cases required an additional pedicled flap to prevent 
further herniation of the cerebrum and our results were consistent with those of previous studies. We preferred to use vascularized nasoseptal flap in cases with high pressure leaks and larger defects, especially those within the sphenoid sinus. Long and wide-based nasoseptal mucosal flaps were necessary in order to seal the defects located within the sinus (20). Importantly, all seven CSF leaks in this series were successfully repaired with use of nasoseptal flaps on the first attempt. In a recent review article, Sharma et al. did not reach a final conclusion on the best available material for endoscopic repair of CSF fistulas (32). However, in our cases, high-pressure and large-diameter leaks needed inlay free fascia grafts and all were held in place by 4.0 vicryl suture at the center of the graft. We believed that placement of additional overlay free abdominal muscle grafts, followed by vascularized pedicled flaps and final application of fibrin glue improved graft adherence and strength of the repair in these cases.

Endoscopic repair of CSF fistulas accounted for nearly 5.4\% of all cases operated via endoscopic endonasal approach in our center (43 endoscopic endonasal approaches in 39 patients). The endonasal endoscopy experience gained in our clinic and the gradual expansion of the routes to different parts of the skull base through EESBA, along with the encouraging initial results on CSF fistula repair, herald an important shift in the selection of surgical options for VMSBL. Our $96 \%$ overall success rate for CSF leak closure via endoscopic endonasal approach practically eliminated the use of transcranial approaches for cases with VMSBL. This abrupt transformation in the surgical management of CSF leak repair conceivably prevented major complications related to traditional transcranial approaches via the frontal lobe, including anosmia, intracranial hemorrhage, cerebral edema, seizures, and memory deficits (4).

The main limitation of our study is relative small sample size with mixed etiologies.

\section{- CONCLUSION}

Similar to previously reported outcomes, this study demonstrated that endoscopic endonasal neurosurgical repair of VMSBL is safe and highly effective, with very low complication rates. EESBA can be a first line surgical treatment option for VMSBL, practically making the transcranial approaches redundant. Furthermore, accompanying ventral midline skull base meningoencephaloceles can adequately be reconstructed via EESBA, regardless of size. Vascularized pedicled nasoseptal flaps can improve closure rates in larger defects and in leaks within the sphenoidal sinus. Successful management of CSF rhinorrhea can be possible with a well executed surgical plan, which should rest on thorough knowledge of the endoscopic anatomy of the skull base.

\section{- ACKNOWLEDGEMENT}

Preparation for publication of this article is partly supported by Turkish Neurosurgical Society.

\section{REFERENCES}

1. Algin O, Hakyemez B, Gokalp G, Ozcan T, Korfali E, Parlak $\mathrm{M}$ : The contribution of 3D-CISS and contrast-enhanced MR cisternography in detecting cerebrospinal fluid leak in patients with rhinorrhoea. Br J Radiol 83(987):225-232, 2010

2. Bedrosian JC, Anand VK, Schwartz TH: The endoscopic endonasal approach to repair of iatrogenic and noniatrogenic cerebrospinal fluid leaks and encephaloceles of the anterior cranial fossa. World Neurosurg 82(6S):S86-94, 2014

3. Bhalodiya $\mathrm{NH}$, Joseph ST: Cerebrospinal fluid rhinorrhea: Endoscopic repair based on a combined diagnostic approach. Indian J Otolaryngol Head Neck Surg 61:120-126, 2009

4. Bibas AG, Skia B, Hickey SA: Transnasal endoscopic repair of cerebrospinal fluid rhinorrhea. Br J Neurosurg 14(1):49-52, 2000

5. Briggs RJ, Wormald PJ: Endoscopic transnasal intradural repair of anterior skull base cerebrospinal fluid fistulae. J Clin Neurosci 11(6):597-599, 2004

6. Cairns $\mathrm{H}$ : Injuries of the frontal and ethmoidal sinuses with special references to cerebrospinal rhinorrhea and aeroceles. J Laryngol Otol 52(9):589-623, 1937

7. Castelnuovo P, Dallan I, Pistochini A, Battaglia P, Locatelli D, Bignami M: Endonasal endoscopic repair of Sternberg's canal cerebrospinal fluid leaks. Laryngoscope 117(2):345-349, 2007

8. Chappity P, Alok T, Rohit V: Endonasal endoscopic approach in management of pediatric CSF rhinorrhoea cases. Indian $\mathrm{J}$ Otolaryngol Head Neck Surg 67(1):88-92, 2015

9. de Almeida JR, Ghotme K, Leong I, Drake J, James AL, Witterick IJ: A new porcine skull base model: Fibrin glue improves strength of cerebrospinal fluid leak repairs. Otolaryngol Head Neck Surg 141(2):184-189, 2009

10. Gassner HG, Ponikau JU, Sherris DA, Kern EB: CSF rhinorrhea: 95 consecutive surgical cases with long term follow-up at the Mayo Clinic. Am J Rhinol 13(6):439-447, 1999

11. Har-el G: What is "spontaneous" cerebrospinal fluid rhinorrhea? Classification of cerebrospinal fluid leaks. Ann Otol Rhinol Laryngol 108(4):323-326, 1999

12. Hegazy HM, Carrau RL, Snyderman CH, Kassam A, Zweig J: Transnasal endoscopic repair of cerebrospinal fluid rhinorrhea: A meta analysis. Laryngoscope 110(7):1166-1172, 2000

13. Hirsch O: Successful closure of cerebrospinal fluid rhinorrhea by endonasal surgery. AMA Arch Otolaryngol 56(1):1-12, 1952

14. Hosemann W, Goede U, Sauer M: Wound healing of mucosal autografts for frontal cerebrospinal fluid leaks clinical and experimental investigations. Rhinology 37(3):108-112, 1999

15. Ismail AS, Costantino PD, Sen C: Transnasal transsphenoidal endoscopic repair of CSF leakage using multilayer acellular dermis. Skull Base 17(2):125-132, 2007

16. Kerr JT, Chu FW, Bayles SW: Cerebrospinal fluid rhinorrhea: Diagnosis and management. Otolaryngol Clin North Am 38(4): 597-611, 2005

17. Lanza DC, O'Brien DA, Kennedy DW: Endoscopic repair of cerebrospinal fluid fistulae and encephaloceles. Laryngoscope 106:1119-1125, 1996 
18. Locatelli D, Rampa F, Acchiardi I, Bignami M, De Bernardi F, Castelnuovo P: Endoscopic endonasal approaches for repair of cerebrospinal fluid leaks: Nine-year experience. Neurosurgery 58(4 Suppl 2):ONS-246-256, 2006

19. Mattox DE, Kennedy DW: Endoscopic management of cerebrospinal fluid leaks and cephaloceles. Laryngoscope 100(8):857-862, 1990

20. McCoul ED, Anand VK, Singh A, Nyquist GG, Schaberg MR, Schwartz TH: Long-term effectiveness of a reconstructive protocol using the nasoseptal flap after endoscopic skull base surgery. World Neurosurg 81(1):136-143, 2014

21. Melo NA, Borges BB, Magliarelli Filho PA, Godoy MD, Pereira LV, Pinna Fde R, Voegels RL: Lateral sphenoid sinus recess cerebrospinal fluid leak: A case series. Eur Arch Otorhinolaryngol 271(9):2587-2594, 2014

22. Nyquist GG, Anand VK, Mehra S, Kacker A, Schwartz TH: Endoscopic endonasal repair of anterior skull base nontraumatic cerebrospinal fluid leaks, meningoceles and encephaloceles. J Neurosurg 113(5):961-966; 2010

23. Nyquist GG, Anand VK, Schwartz TH: Endoscopic management of cerebrospinal fluid rhinorrhea. Operative Techniques in Otolaryngology 22(3): 229-231, 2011

24. Papay FA, Maggiano H, Dominquez S, Hassenbusch SJ, Levine HL, Lavertu P: Rigid endoscopic repair of paranasal sinus cerebrospinal fluid fistulas. Laryngoscope 99(11):11951201, 1989

25. Pappas DG, Hammerschlag PE, Hammerschlag M: Cerebrospinal fluid rhinorrhea and recurrent meningitis. Clin Infect Dis 17(3):364-368, 1993

26. Presutti L, Mattioli F, Villari D, Marchioni D, Alicandri-Ciufelli M: Transnasal endoscopic treatment of cerebrospinal fluid leak: 17 years' experience. Acta Otorhinolaryngol Ital 29(4):191196, 2009

27. Psaltis AJ, Schlosser RJ, Banks CA, Yawn J, Soler ZM: A systematic review of the endoscopic repair of cerebrospinal fluid leaks. Otolaryngol Head Neck Surg 147(2): 196-203, 2012
28. Saafan ME, Albirmawy OA, Tomoum MO: Sandwich grafting technique for endoscopic endonasal repair of cerebrospinal fluid rhinorrhoea. Eur Arch Otorhinolaryngol 271(5):10731079, 2014

29. Schick B, lbing R, Brors D, Draf W: Long-term study of endonasal duraplasty and review of the literature. Ann Otol Rhinol Laryngol 110(2):142-147, 2001

30. Schmerber S, Righini C, Lavielle JP, Passagia JG, Reyt E: Endonasal endoscopic closure of cerebrospinal fluid rhinorrhea. Skull Base 11(1):47-58, 2001

31. Sciarretta V, Mazzatenta D, Ciarpaglini R, Pasquini E, Farneti G, Frank G: Surgical repair of persisting CSF leaks following standard or extended endoscopic transsphenoidal surgery for pituitary tumor. Minim Invasive Neurosurg 53(2):55-59, 2010

32. Sharma SD, Kumar G, Bal J, Eweiss A: Endoscopic repair of cerebrospinal fluid rhinorrhoea. Eur Ann Otorhinolaryngol Head Neck Dis 133(3):187-190, 2016

33. Spetzler RF, Wilson CB: Management of recurrent CSF rhinorrhea of the middle and posterior fossa. J Neurosurg 49(3):393-397, 1978

34. Stankiewicz JA: Cerebrospinal fluid fistula and endoscopic sinus surgery. Laryngoscope 101(3):250-256, 1991

35. Tabaee A, Anand VK, Cappabianca P, Stamm A, Esposito F, Schwartz TH: Endoscopic management of spontaneous meningoencephalocele of the lateral sphenoid sinus. J Neurosurg 112(5):1070-1077, 2010

36. Wang EW, Vandergrift WA 3rd, Schlosser RJ: Spontaneous CSF Leaks. Otolaryngol Clin North Am 44(4):845-856, 2011

37. Wigand ME: Transnasal ethmoidectomy under endoscopic control. Rhinology 19(1):7-15, 1981

38. Wise SK, Schlosser RJ: Evaluation of spontaneous nasal cerebrospinal fluid leaks. Curr Opin Otolaryngol Head Neck Surg 15(1):28-34, 2007 